\title{
Potencial energético dos resíduos da castanha do Brasil (Bertholletia excelsa H.B.K.) para produção de carvão ativado
}

Energy potential of waste from Brazil nut (Bertholletia excelsa H.B.K.) for production of activated carbon

Potencial energético de residuos de castaño brasileño (Bertholletia excelsa H.B.K.) para la producción de carbón activado

Recebido: 05/02/2021 | Revisado: 16/02/2021 | Aceito: 19/02/2021 | Publicado: 27/02/2021

Cristiane Daliassi Ramos de Souza
ORCID: https://orcid.org/0000-0003-3857-9719
Universidade Federal do Amazonas, Brasil
E-mail: cdaliassi@ @fam.edu.br
Kleyner de Carvalho da Silva
ORCID: https://orcid.org/0000-0003-0246-5322
Universidade Federal do Amazonas, Brasil
E-mail: kleynersilva.kcs@ @mail.com

\section{Resumo}

Para escolher o processo mais eficiente de geração de energia é necessário conhecer as propriedades químicas e térmicas da biomassa. As cascas e os ouriços da castanha do Brasil (Bertholletia excelsa H.B.K.), geralmente tratados como resíduos, têm despertado interesse de pesquisadores do setor energético devido as suas propriedades carburantes. Este trabalho visa avaliar as propriedades energéticas das cascas da semente e dos ouriços da castanha do Brasil para utilizá-los na produção de adsorventes. Os ensaios físico-químicos e térmicos das biomassas foram investigados pelas técnicas de Análise Imediata (umidade, cinzas, voláteis e carbono fixo), Análise Elementar, Poder Calorífico Superior (PCS), Poder Calorífico Inferior (PCI) e Termogravimetria (TG/DTG). Os adsorventes, CA-1 (cascas) e CA-2 (ouriço), foram produzidos por ativação química com $\mathrm{ZnCl}_{2}$ seguida de carbonização à $500^{\circ} \mathrm{C}$ por 90 min. Os resultados da caracterização para as biomassas mostraram umidade entre $8-9 \%$, cinzas abaixo de $2,8 \%$, elevados teores de voláteis (85\%), PCI e PCS próximos, na faixa entre $15-17 \mathrm{MJ} / \mathrm{kg}$. O rendimento do processo de obtenção dos carvões ativados $(\sim 74 \%)$ foram superiores a literatura. A avaliação dos adsorventes quanto ao processo de adsorção do azul de metileno mostrou-se eficiente, sendo que o carvão ativado produzido das cascas apresentou uma maior capacidade adsortiva quando comparado ao adsorvente produzido a partir dos ouriços. Por fim, as biomassas demonstraram um bom potencial energético e como uma alternativa ao carvão ativado comercial, promovendo o aproveitamento dos resíduos e contribuindo como uma possível solução na busca por materiais precursores de baixo custo para a produção de adsorventes.

Palavras-chave: Castanha do brasil; Biomassa residual; Propriedades energéticas; Carvão ativado; Adsorção.

\begin{abstract}
To choose the most efficient energy generation process, it is necessary to know the properties chemical and thermal effects of biomass. The shells and hedgehogs of Brazil nut (Bertholletia excelsa H.B.K.), generally treated as waste, they have aroused the interest of researchers in the energy sector due to their fuel properties. This work aims to evaluate the energetic properties of seed shells and hedgehogs Brazil nut to use them in the production of adsorbents. The physical-chemical and thermal tests of the biomasses were investigated by the techniques of Proximate Analysis (moisture, ash, volatiles and fixed carbon), Ultimate Analysis, High Heating Value (HHV), Lower Heating Value (LHV) and Thermogravimetry (TG/DTG). The adsorbents, CA-1 (shells) and CA-2 (hedgehog), were produced by chemical activation with $\mathrm{ZnCl}_{2}$ followed by carbonization at $500^{\circ} \mathrm{C}$ for $90 \mathrm{~min}$. The results of the characterization for the biomasses showed moisture between $8-9 \%$, ash below $2.8 \%$, and high volatiles $(85 \%)$ LHV and HHV nearby, in the range between $15-17 \mathrm{MJ} / \mathrm{kg}$. The yield of the process of obtaining activated carbon $(\sim 74 \%)$ was higher than the literature. The evaluation of the adsorbents regarding the methylene blue adsorption process proved to be efficient, activated carbon produced from the shells showed a greater adsorptive capacity when compared to the adsorbent produced from hedgehogs. Finally, the biomasses demonstrated a good energy potential and as an alternative to commercial activated carbon, promoting the use of waste and contributing as a possible solution in the search for lowcost precursor materials for adsorbents production.
\end{abstract}

Keywords: Brazil nut; Residual biomass; Energetic properties; Activated carbon; Adsorption. 


\section{Resumen}

Para elegir el proceso de generación de energía más eficiente, es necesario conocer las propiedades químicas y térmicas de la biomasa. Las cáscaras y erizos de castaña (Bertholletia excelsa H.B.K.), generalmente tratadas como residuos, han despertado el interés de investigadores del sector energético por sus propiedades combustibles. Este trabajo tiene como objetivo evaluar las propiedades energéticas de las cáscaras de semillas y erizos de castaña para utilizarlos en la producción de adsorbentes. Los ensayos fisicoquímicos y térmicos de las biomasas se investigaron mediante las técnicas de Análisis Inmediato (humedad, cenizas, volátiles y carbono fijo), Análisis Elemental, Poder Calorífico Superior (PCS), Poder Calorífico Inferior (PCI) y Termogravimetría (TG/DTG). Los adsorbentes, CA-1 (conchas) y CA-2 (erizo), se produjeron mediante activación química con $\mathrm{ZnCl}_{2}$ seguida de carbonización a $500^{\circ} \mathrm{C}$ durante $90 \mathrm{~min}$. Los resultados de la caracterización para las biomasas mostraron humedad entre 8-9\%, cenizas por debajo de $2.8 \%$, altos niveles de volátiles (85\%) PCI y PCS cercanos, en el rango de 15-17 MJ/kg). El rendimiento del proceso de obtención de carbón activado ( $74 \%)$ fue superior al de la literatura. La evaluación de adsorbentes con respecto al proceso de adsorción con azul de metileno resultó ser eficiente, y el carbón activado producido a partir de las conchas mostró una mayor capacidad adsorbente en comparación con el adsorbente producido a partir de erizos. Finalmente, las biomasas mostraron un buen potencial energético y como alternativa al carbón activado comercial, promoviendo el uso de residuos y contribuyendo como una posible solución en la búsqueda de materiales precursores de bajo costo para la producción de adsorbentes.

Palabras clave: Nueces de brasil; Biomasa residual; Propiedades energéticas; Carbón activado; Adsorción.

\section{Introdução}

O uso da biomassa possui grande relevância no Brasil devido à intensa atividade agrícola e às grandes quantidades de resíduos agroindustriais gerados. Com isso muitos pesquisadores estão voltados para resolver o problema da redução do volume de resíduos de biomassa que, apesar de serem biodegradáveis, necessitam de um tempo mínimo para serem mineralizados. Desta forma, vários estudos têm avaliado a possibilidade de aplicação destes resíduos como fonte de energia renovável (Couto, Couto, Watzlawick, \& Câmara, 2004; Marafon et al., 2016; Saini, Saini, \& Tewari, 2015; Scatolino et al., 2018).

Algumas cidades do norte do Brasil, localizadas na Amazônia brasileira, baseiam sua economia na agricultura, na fruticultura e no extrativismo. Frutas como cupuaçu, açaí, patoá, castanha do Brasil são responsáveis por gerar renda a essas regiões e um grande volume de resíduos que são desperdiçados ou mal aproveitados. Este material descartado, poderia ser aproveitado de diversas formas, inclusive na geração de energia para cidades do interior do Amazonas com sistemas elétricos isolados da rede nacional, onde a maioria das usinas termelétricas são abastecidas a óleo diesel (Souza \& Trindade, 2015).

Uma possibilidade para o aproveitamento desses resíduos é a produção de carvão ativado, um material que tem alta capacidade de adsorção, possibilitando sua utilização na recuperação de produtos químicos, na remoção de compostos orgânicos e metais, na remoção de contaminantes presentes em meio aquoso, suporte para catalisadores, processos de tratamento de água, entre outros (Reza et al., 2020; Ghasemi et al., 2016; Nobre et al., 2015).

Dentre os diversos resíduos, destaca-se uma biomassa residual com potencial energético na Amazônia, a Castanha do Brasil (Bertholletia excelsa H.B.K.), pois tem ampla distribuição na floresta Amazônica (Ferreira, Silveira, Lucien, \& Amaral, 2009). É também conhecida como castanha verdadeira, castanheira e castanha do Pará. A planta é de grande porte, podendo medir até $60 \mathrm{~m}$ de altura, com diâmetro de 100 a $180 \mathrm{~cm}$ (Costa, Castro, Wandelli, Coral, \& Souza, 2009).

Em geral, apenas as amêndoas são aproveitadas por serem comestíveis, formando todo o resto do fruto, cerca de $90 \%$, os resíduos. A parte externa do fruto, denominada de ouriço, que constitui o pericarpo; e a interna, que são as cascas da semente (ou tegumentos), de onde são extraídas as amêndoas (Bouvie, Borella, Porto, Silva, \& Leonel, 2016). De acordo com Oliveira e Lobo (2002), o fruto da castanha do Brasil tem, em geral 2,4 kg, no qual aproximadamente 1/3 de seu peso é constituído pelas sementes. Logo, para cada fruto coletado, tem-se, em média, 1,6 kg de resíduo, que pode ser aproveitado.

Portanto, o aproveitamento dos resíduos da agroindústria para a produção de carvão ativado é de suma importância, uma vez que este produto poderá agregar valor a uma parte do material que normalmente é descartado. Além disso, os 
adsorventes produzidos podem trazer benefícios ambientais em diversos processos industriais (Januszewicz, Kazimierski, Klein, Karda's, \& Łuczak, 2020; Leandro et al., 2019; Hameed, Muthirulan, \& Meenakshi, 2017; Melo et al., 2015).

\section{Metodologia}

\subsection{Coleta e Pré-Tratamento dos Resíduos}

Os frutos inteiros da Castanha do Brasil foram fornecidos por um extrativista de Careiro da Várzea, município do Amazonas, para o projeto "Produção de biodiesel utilizando biomassa regional da Amazônia" (Fernandes \& Souza, 2016). Os resíduos (ouriços e cascas da semente) do processo de obtenção do biodiesel foram colocados em sacos plásticos e armazenados na geladeira do Laboratório de Controle de Qualidade do DEQ/UFAM.

Inicialmente, os materiais foram submetidos a secagem em estufa com circulação de ar (LimaTec, modelo LT96EC), a $105^{\circ} \mathrm{C}$ por $2 \mathrm{~h}$, para retirada de umidade. Posteriormente, foram encaminhados para moagem, utilizando um moinho de facas (Marconi; modelo MA048), gerando diferentes faixas granulométricas para os materiais.

A etapa seguinte foi a análise granulométrica, a qual permite identificar o tamanho e a distribuição dos grãos. Cada resíduo foi submetido a classificação utilizando peneiras (TPL) com abertura de $297 \mu \mathrm{m}$.

\subsection{Caracterização Energética dos Resíduos}

A Análise Imediata compreende os ensaios para quantificar os teores de umidade, cinzas, materiais voláteis e carbono fixo. Os experimentos foram baseados na NBR 8112 (ABNT, 1986) e realizados em triplicata no Laboratório de Controle de Qualidade do DEQ/UFAM.

A composição elementar (C, H, O) foi calculada pelas equações de (1) a (3) desenvolvidas por Shen, Zhu, Liu, Zhang, e Tan (2010) que utilizam as relações empíricas com base nos dados experimentais dos teores de materiais voláteis (V), carbono fixo (CF) e cinzas (CZ) da biomassa avaliada.

$$
\begin{aligned}
& C=(0,635 * C F)+(0,460 * V)-(0,095 * C Z) \\
& H=(0,059 * C F)+(0,060 * V)+(0,010 * C Z) \\
& O=(0,340 * C F)+(0,469 * V)-(0,023 * C Z)
\end{aligned}
$$

A determinação do Poder Calorífico Superior (PCS), em MJ/kg, foi realizada pela utilização da equação (4) desenvolvida por Parikh, Channiwala e Ghosal (2005), onde relacionam-se os dados experimentais da análise imediata. Já o Poder Calorífico Inferior (PCI), em kcal/kg, foi calculado pela equação (5) proposta por Sanchez (2010) que é dependente do teor de hidrogênio do material.

$$
\begin{aligned}
& P C S=(0,3536 * \mathrm{CF})+(0,1559 * \mathrm{MV})-(0,0078 * \mathrm{CZ}) \\
& P C I=(\mathrm{PCS}-50,68 * \mathrm{H} * 4,187)
\end{aligned}
$$


A análise termogravimétrica (TG/DTG) foi realizada no Laboratório de ensaios de materiais da FT/UFAM utilizando o equipamento SDT Q600 (TA Instruments), com fluxo de $\mathrm{N}_{2}$ de $30 \mathrm{~mL} / \mathrm{min}$ e razão de aquecimento de $10^{\circ} \mathrm{C} / \mathrm{min}$, da temperatura ambiente até $1000^{\circ} \mathrm{C}$.

\subsection{Produção do Carvão Ativado}

Primeiramente, para o processo de obtenção do carvão ativado das cascas da semente (CA-1) e dos ouriços (CA-2), foi realizada a ativação química. Cada resíduo foi impregnado com $\mathrm{ZnCl}_{2}$ (Nuclear; $97 \%$ ) na proporção mássica resíduo: $\mathrm{ZnCl}_{2}$ de 1:2, seguindo a metodologia de Lillo-Ródenas, Juan-Juan, Cazorla-Amorós e Linares-Solano (2004). As misturas ficaram sob agitação por $1 \mathrm{~h}$ com posterior repouso de $2 \mathrm{~h}$. Em seguida foram colocadas na estufa para secagem a $105^{\circ} \mathrm{C}$ por $72 \mathrm{~h}$.

Para a etapa de carbonização cerca de $15 \mathrm{~g}$ de cada mistura seca foi colocada em forno mufla a $500^{\circ} \mathrm{C}$, $\operatorname{com}$ taxa de aquecimento de $5^{\circ} \mathrm{C} / \mathrm{min}$ e tempo de residência de $90 \mathrm{~min}$, para remoção de materiais voláteis e aumento no teor de carbono. Finalizado o processo, o CA-1 e o CA-2 foram lavados com água destilada até pH neutro, para a remoção do agente ativante e desobstrução dos poros formados (Yagub, Sen, \& Ang, 2012). A massa final foi registrada para o cálculo do rendimento.

De acordo com Himmelblau e Riggs (2014), o balanço de massa fundamenta-se no princípio de conservação da massa. Logo, foi possível obter dados que representam o rendimento do processo. Com isso, para cada carvão ativado obtido foi efetuada a determinação do rendimento $(R)$ pela equação (6).

$$
\mathrm{R}=\frac{\mathrm{m}_{\mathrm{f}}(\mathrm{CA})}{\mathrm{m}_{\mathrm{i}}(\mathrm{mistura})} \times 100
$$

onde, "mf (CA)" é a massa do carvão ativado obtida após o processo de carbonização; "mi (mistura)" é a massa da mistura antes da carbonização, ambas em base seca.

\subsection{Ensaios de Adsorção}

Foram realizados estudos variando o tempo de contato e a temperatura das soluções, com o objetivo de avaliar o efeito destes parâmetros no processo de adsorção. Os ensaios foram realizados em batelada para cada adsorvente produzido: CA-1 e CA-2.

Em tubos de ensaio de $100 \mathrm{~mL}$, adicionaram-se 0,1 g de adsorvente e $10 \mathrm{~mL}$ da solução do corante azul de metileno com concentrações de $(2 ; 5 ; 7 ;$ e $10 \mathrm{mg} / \mathrm{L})$ a $25^{\circ} \mathrm{C}$, sob agitação constante por 60 min e posterior repouso de $24 \mathrm{~h}$. Em seguida, alíquotas da solução foram centrifugadas (Kacil, modelo CE01), $3000 \mathrm{rpm}$ por $10 \mathrm{~min}$. A análise da concentração final do corante foi determinada por espectroscopia UV-Visível (Coleman-35D) no comprimento de onda de maior absorção do corante $(664 \mathrm{~nm})$.

\subsubsection{Efeito do Tempo de Contato}

Para avaliar o parâmetro do tempo no processo de adsorção do corante azul de metileno para o CA-1 e CA-2, durante os instantes de tempo de 10, 20, 30, 40, 50 e 60 min, foram realizadas coletas de amostras das soluções com as concentrações de estudo (2; 5; 7; e $10 \mathrm{mg} / \mathrm{L})$. O perfil de adsorção foi determinado por espectrofotometria UV-Visível (664 nm). 


\subsubsection{Efeito da Temperatura}

Para obtenção dos dados experimentais nos ensaios de adsorção, novamente utilizou-se a proporção de $0,1 \mathrm{~g}$ de adsorvente para $10 \mathrm{~mL}$ de corante. Nos tubos 1,2 e 3 foram ajustadas as temperaturas para $25^{\circ} \mathrm{C}, 35^{\circ} \mathrm{C}$ e $45^{\circ} \mathrm{C}$, respectivamente, durante $60 \mathrm{~min}$. A absorbância foi determinada por espectrofotometria UV-Visível. O procedimento foi realizado para as faixas de concentrações em estudo $(2 ; 5 ; 7 ;$ e $10 \mathrm{mg} / \mathrm{L})$.

\section{Resultados e Discussão}

\subsection{Análise Imediata}

Para que resíduos agroindustriais possam ser utilizados para geração de energia, características da biomassa devem ser investigadas e controladas, como os teores de umidade, voláteis, cinzas e carbono fixo. Os resultados da análise imediata dos resíduos, com granulometria de $297 \mu \mathrm{m}$, são apresentados na Tabela 1.

Tabela 1. Análise Imediata dos resíduos da Castanha do Brasil (Bertholletia excelsa H.B.K.).

\begin{tabular}{l|c|c}
\hline \multicolumn{1}{c|}{ Análise Imediata } & Cascas da Semente & Ouriços \\
\hline Umidade (\%) & $8,51 \pm 0,23^{*}$ & $9,05 \pm 0,82^{*}$ \\
\hline Cinzas (\%) & $2,56 \pm 0,38^{*}$ & $2,74 \pm 0,27^{*}$ \\
\hline Voláteis (\%) & $90,81 \pm 1,51^{*}$ & $88,04 \pm 0,56^{*}$ \\
\hline Carbono Fixo (\%) & $6,63 \pm 1,54^{*}$ & $9,22 \pm 1,05^{*}$ \\
\hline
\end{tabular}

* Desvio Padrão. Fonte: Autores.

O teor de umidade encontrado nos resíduos ficou abaixo de 10\%, corroborando com os resultados de Vassilev, Baxter, Vassileva e Andersen (2013), assim como os de Nascimento (2012). A umidade interfere diretamente no poder calorífico, pois quanto mais elevada maior será o gasto energético utilizado no processo de combustão do material e menor será a energia liberada pela reação endotérmica (Everard, Mcdonnell, \& Fagan, 2012).

Quanto ao teor de cinzas, Brand (2010) cita que é desejável que o carvão ativado apresente valores baixos, uma vez que teores elevados de minerais contribuem para redução do poder calorífico. $\mathrm{O}$ valor médio encontrado para os ouriços $(2,74 \%)$ e para as cascas $(2,56 \%)$ estão próximos aos dados da literatura para biomassas vegetais (Nogueira, Ruffato, Carneiro, Pires, \& Alvares, 2014; Nascimento, 2012).

Assim como a umidade, o teor de cinzas elevado interfere no resultado do poder calorífico, pois causa perda de energia e prejudica a troca térmica. Além disso, as cinzas são consideradas materiais abrasivos e que podem causar danos nos equipamentos, como corrosões (Vieira, 2013; Klautau, 2008). Desta forma, a baixa quantidade de cinzas apresentada pelas biomassas é um ótimo indicativo para sua aplicabilidade para em processos energéticos.

Os materiais voláteis referem-se aos gases desprendidos da biomassa durante a carbonização (Nogueira et al., 2014). O valor médio encontrado para os resíduos ficou acima de $86 \%$, corroborando para o alto poder de ignição, uma vez que apresenta resultados superiores aos encontrados na literatura (Nascimento, 2012; Santos, \& Júnior, 2016). De acordo com Klautau (2008) quanto mais elevado o teor de voláteis maior será a reatividade e consequentemente a ignição.

Já o valor médio de carbono fixo apresentou valores na faixa de 6-10\%, estes resultados foram próximos aos estudos de Nascimento (2012) e de Oliveira e Lobo (2002). Vale ressaltar que a quantidade de carbono fixo relaciona os valores de cinzas e voláteis presentes na biomassa. 


\subsection{Poder Calorífico}

O Poder Calorífico, seja ele superior (PCS) ou Inferior (PCI), influencia na escolha do combustível. O PCS representa a energia liberada pela combustão tendo toda a água resultante na fase líquida e o PCI representa o calor liberado pela combustão estando toda a água resultante no estado gasoso. A Figura 1 apresenta uma comparação dos resultados do PCS e PCI calculados para as biomassas avaliadas.

Figura 1. Comparação entre os resultados do PCS e do PCI para o ouriço e para as cascas da Castanha do Brasil (Bertholletia excelsa H.B.K.).

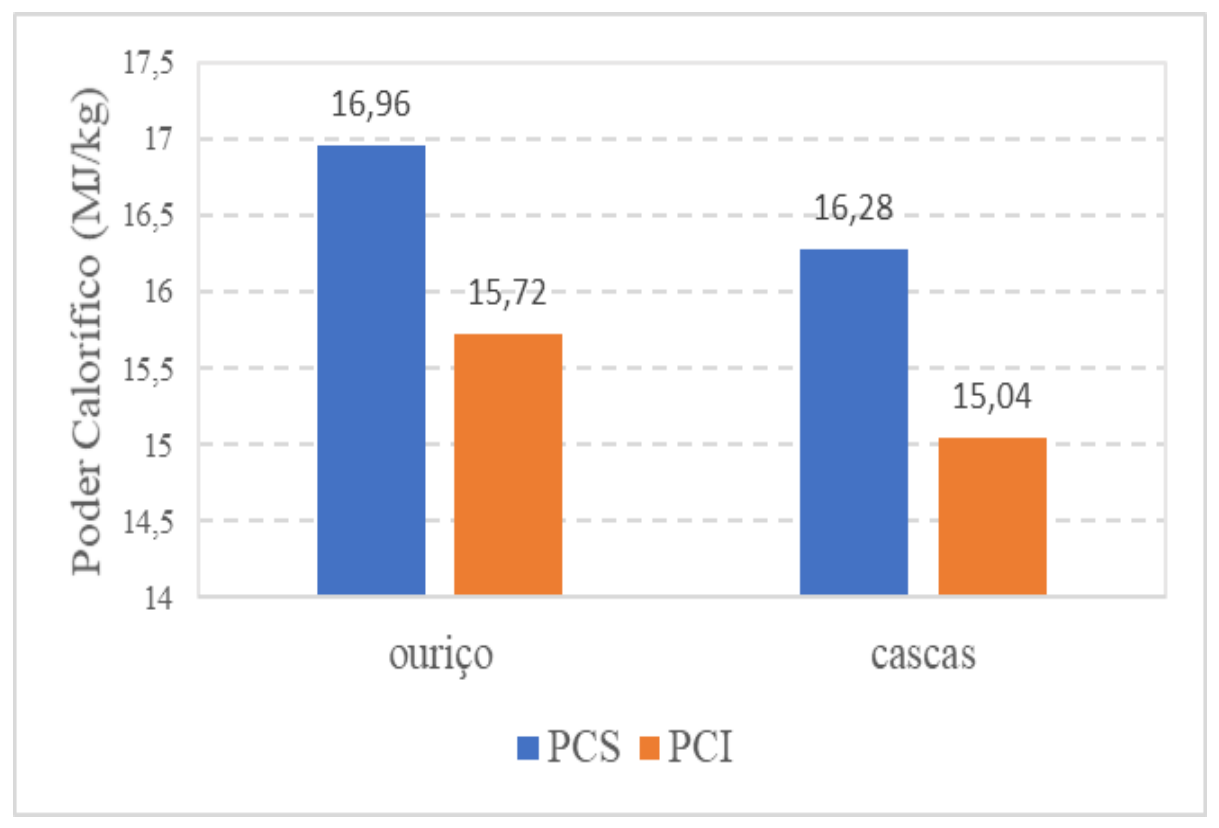

Fonte: Autores.

O PCS, obtido através da equação (4), foi de 16,28 MJ/kg e 16,96 MJ/kg para as cascas e ouriços, respectivamente. Os resultados mostram uma pequena superioridade do PCS para o ouriço, ocasionado pela relação direta com o teor de carbono fixo, e inversamente proporcional ao teor de voláteis, conforme encontrado por meio da análise imediata.

Já o PCI, obtido pela correlação matemática apresentada na equação (5), foram encontrados valores próximos, porém menores que o PCS para ambos os resíduos avaliados. De acordo com Nascimento (2012), o PCI é cerca de 10 a $20 \%$ menor que o PCS, pois não considera o calor latente de condensação do vapor da água presente nos produtos da combustão. No presente trabalho, o PCI não ultrapassou os $10 \%$, o que comprova esta afirmação, entretanto o teor de umidade influencia na maior parte das vezes este parâmetro.

\subsection{Análise Elementar}

A determinação da composição elementar de uma biomassa influencia diretamente no seu poder calorífico, pois a maior presença de Carbono e Hidrogênio aumenta o PCS, devido as ligações carbono-hidrogênio serem mais energéticas do que as ligações com o oxigênio. Os resultados da análise elementar, determinados através das equações (1) a (3) e as razões atômicas $\mathrm{O} / \mathrm{C}$ e H/C, para os resíduos são apresentados na Tabela 2. 
Tabela 2. Análise elementar e razões atômicas O/C e H/C dos resíduos da Castanha do Brasil (Bertholletia excelsa H.B.K.).

\begin{tabular}{l|c|c}
\hline \multicolumn{1}{c|}{ Análise Elementar } & Cascas da Semente & Ouriços \\
\hline Carbono (\%) & 45,74 & 46,09 \\
\hline Hidrogênio (\%) & 5,86 & 5,85 \\
\hline Oxigênio (\%) & 44,78 & 44,36 \\
\hline O/C & 0,98 & 0,96 \\
\hline H/C & 0,13 & 0,13 \\
\hline
\end{tabular}

Fonte: Autores.

Os principais componentes da biomassa são carbono, oxigênio, hidrogênio e nitrogênio (Vassilev, Baxter, Vassileva, \& Andersen, 2013). A Tabela 2 mostra que o teor de carbono para o ouriço foi um pouco superior ao da casca da semente, por outro lado o teor de oxigênio foi ligeiramente menor para o ouriço. Desta forma, a relação O/C para a casca da semente $(0,98)$ foi maior do que a do ouriço $(0,96)$.

Já para a relação $\mathrm{H} / \mathrm{C}$, as biomassas apresentaram resultados iguais $(0,13)$. Por possuírem a mesma relação H/C, o que fez com que o ouriço tivesse um maior poder calorífico em relação a casca da semente foi a pequena redução no teor de oxigênio (Mckendry, 2015; Vassilev et al., 2013).

A decomposição térmica durante a carbonização para as cascas e para os ouriços da Castanha do Brasil são mostradas nas curvas termogravimétricas da Figura 2, as quais fornecem correlações entre a perda de massa do resíduo analisado e a temperatura da carbonização.

De acordo com a Figura 2 é observado a ocorrência de três eventos de perdas de massas tanto para as cascas quanto para os ouriços. A primeira perda de massa ocorre entre 25 e $150^{\circ} \mathrm{C}$, é referente as moléculas de água fracamente ligadas (umidade), e apresentou cerca de 3\% para as cascas e de 5\% para os ouriços. No segundo evento, observa-se a maior perda de massa relacionada a decomposição da hemicelulose e da celulose, na faixa entre 200 a $600^{\circ} \mathrm{C}(95 \%$-cascas; $90 \%$-ouriço).

Figura 2. Curvas TG/DTG dos resíduos investigados.

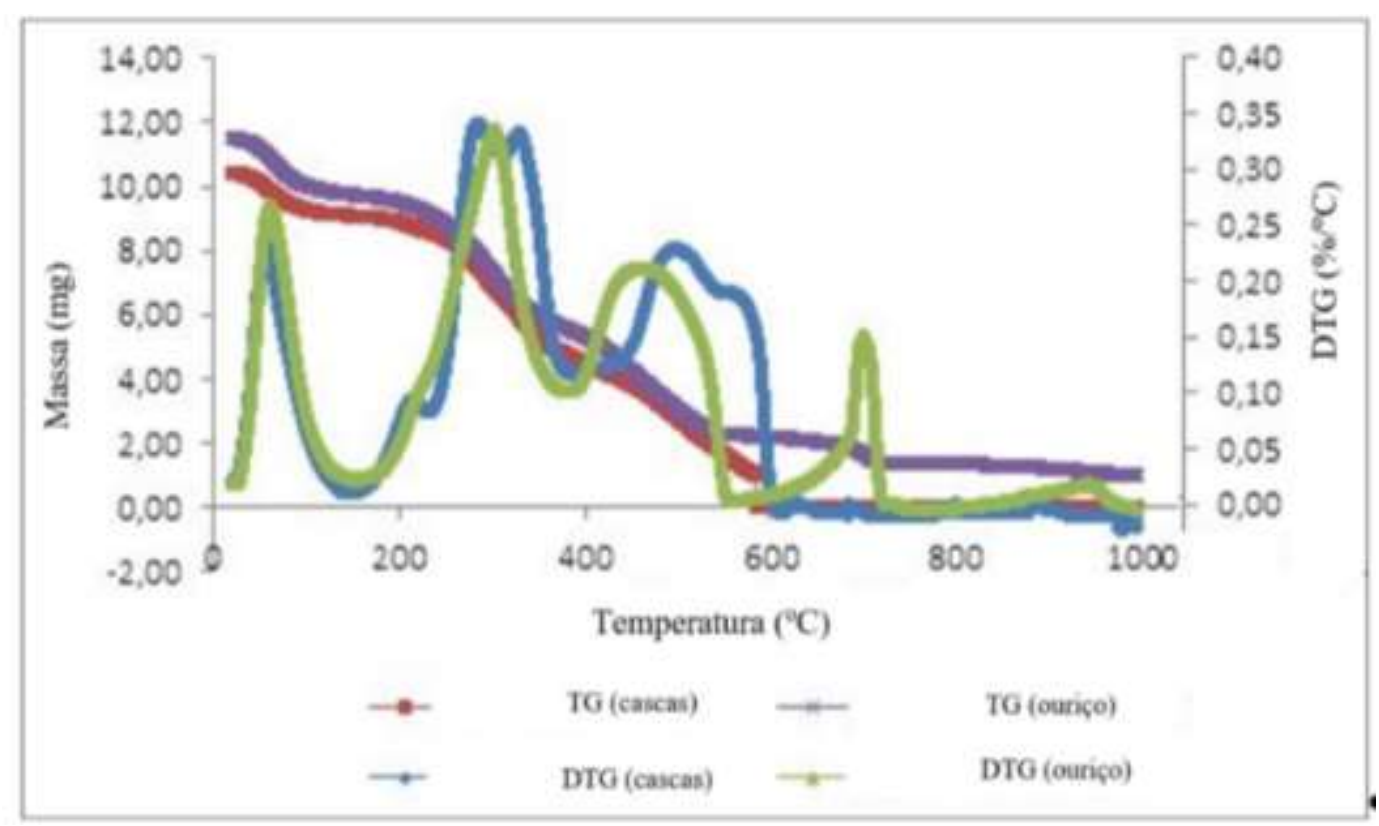

Fonte: Autores. 
$\mathrm{Na}$ faixa de temperatura entre 200 à $390^{\circ} \mathrm{C}$, corresponde a primeira carbonização, com a eliminação dos compostos voláteis, relacionada a decomposição da hemicelulose. Com o aumento da temperatura ocorre a decomposição da celulose, confirmada pela DTG para os dois resíduos com os picos em aproximadamente $300^{\circ} \mathrm{C}$ e $460^{\circ} \mathrm{C}$. O terceiro evento ocorre entre 580 a $770^{\circ} \mathrm{C}$ e corresponde a carbonização final da lignina. Desta forma, a perda de massa nas temperaturas acima de $900^{\circ} \mathrm{C}$ está relacionada à resíduos inorgânicos representando o teor de cinzas, evidenciando tratar-se de biomassa rica em carbono, com elevado potencial para produção de CA (2\%-cascas; 5\%-ouriço) (Pereira, Nobre, \& Bianchi, 2019).

A Figura 3 apresenta os resultados do rendimento gravimétrico do processo de produção do carvão ativado a partir dos resíduos, CA-1 e CA-2.

Figura 3. Rendimento do processo de carbonização a $500^{\circ} \mathrm{C}$ por $90 \mathrm{~min}$ para o CA-1 (cascas) e para o CA-2 (ouriço).

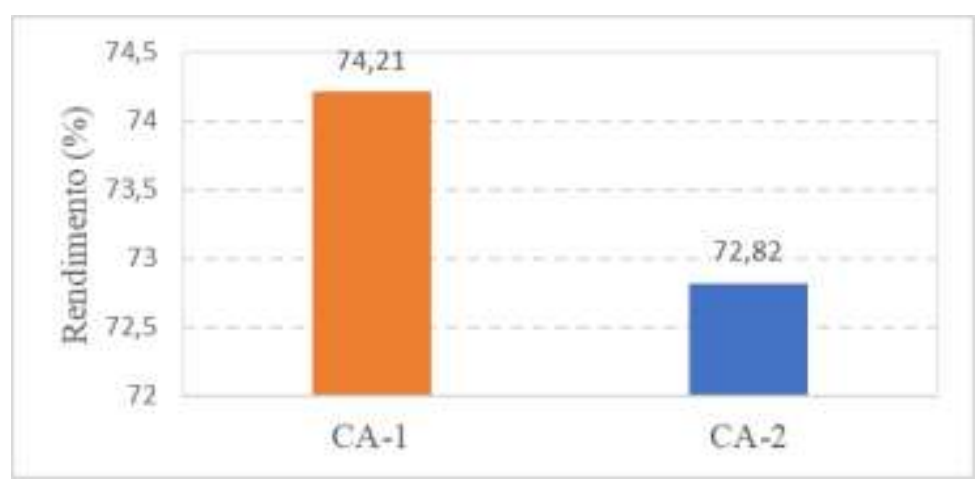

Fonte: Autores.

Uma análise comparativa dos resultados demostra que foi produzido um maior percentual de carvão em relação aos obtidos na literatura (Pereira, Nobre, \& Bianchi, 2019; Carmona et al., 2017; Melo et al., 2015).

\subsection{Efeito do Tempo de Contato na Adsorção do Azul de Metileno}

Nas Figuras de 4 e 5 são apresentadas as dependências da concentração do corante em função do tempo de contato com os adsorventes, CA-1 (cascas) e CA-2 (ouriço).

Figura 4. Concentração de $2 \mathrm{mg} / \mathrm{L}$ (A) e $5 \mathrm{mg} / \mathrm{L}$ (B) do azul de metileno.
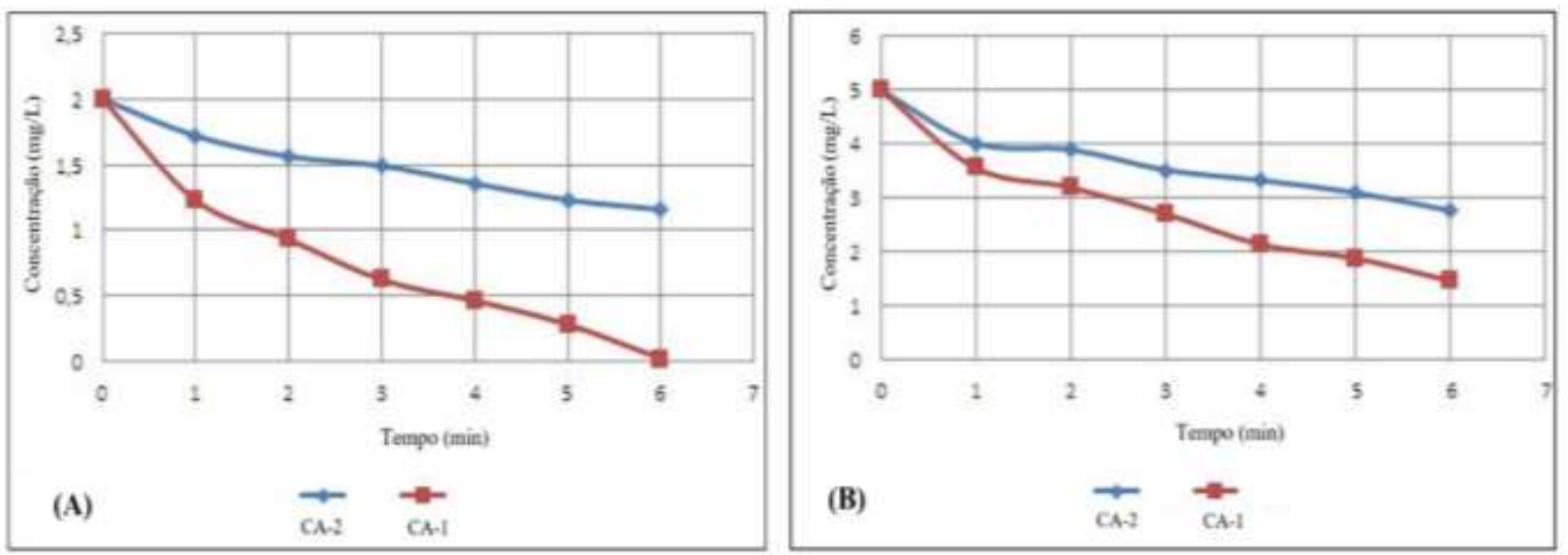

Fonte: Autores. 
Figura 5. Concentração de $7 \mathrm{mg} / \mathrm{L}$ (A) e $10 \mathrm{mg} / \mathrm{L}$ (B) do azul de metileno.
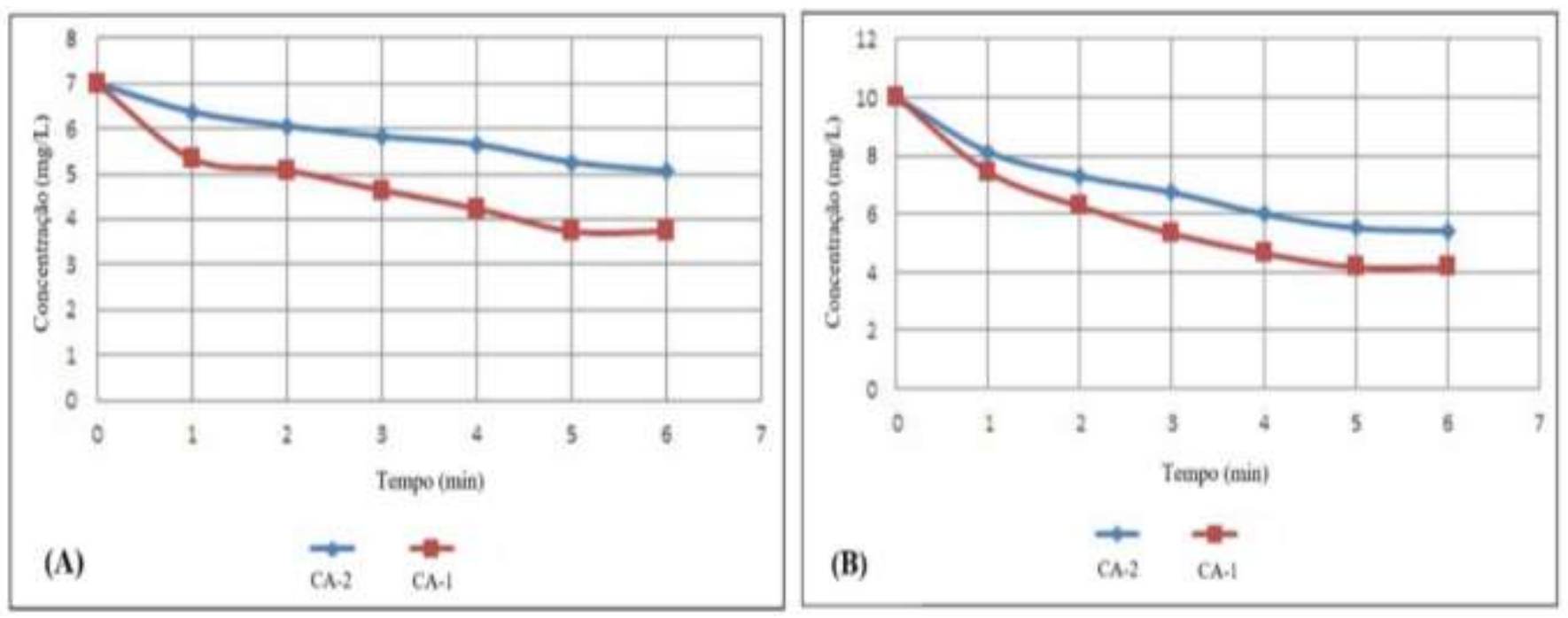

Fonte: Autores.

Como pode ser visto na Figura 4A, para o CA-1 a remoção de corante ocorreu com eficiência de aproximadamente 100\% em apenas 60 min. A amostra CA-2, por sua vez, apresentou em torno de 60\% de eficiência de remoção para o mesmo tempo de contato.

Pelas Figuras 4B, 5A e 5B observam-se que, tanto para CA-1 quanto para CA-2, a remoção do adsorbato aumentou com o tempo de contato no adsorvente com uma tendência ao equilíbrio entre 50 e $60 \mathrm{~min}$. Verificou-se também que durante os primeiros 30 min ocorreu uma elevada remoção do corante, demonstrando uma maior capacidade de adsorção para o CA-1 em todas as concentrações avaliadas. A remoção rápida do adsorbato e o alcance do equilíbrio em um período curto são indicações que os adsorventes são eficientes.

A velocidade em que ocorre a adsorção está relacionada com a quantidade de sítios ativos disponíveis na superfície do carvão e ao menor/maior impedimento estereoquímico para as moléculas de corante que se aproximam, seguidos de um processo mais lento após os sítios terem sidos preenchidos (Albadarin et al., 2017).

\subsection{Efeito da Temperatura na Adsorção do Azul de Metileno}

A temperatura é uma das propriedades que mais influenciam nas relações de equilíbrio, pois atua sobre o poder de agitação das moléculas, interferindo diretamente nas forças de contato e repulsão entre o adsorvato e o adsorvente (Marin et al., 2015). A Figura 6 mostra a influência da temperatura na adsorção do corante, para as quatro concentrações avaliadas, utilizando o CA-1 e o CA-2 produzidos a partir dos resíduos.

Pode-se verificar que com o aumento da temperatura ocorreu uma diminuição da concentração do corante na solução em relação a inicial, proporcionando uma maior capacidade adsortiva para os materiais. Esse comportamento foi observado para os dois carvões, CA-1 e CA-2. Os resultados mostram indícios de que estão seguindo uma tendência no comportamento da capacidade adsortiva com o aumento da temperatura, indicando que o processo de adsorção do azul de metileno pode ser endotérmico (Patias et al., 2015). 
Figura 6. Influência da temperatura na remoção do corante nas concentrações: (a) $2 \mathrm{mg} / \mathrm{L}$, (b) 5 mg/L, (c) 7 mg/L e (d) 10 mg/L, utilizando o CA-1 (A) e CA-2 (B).
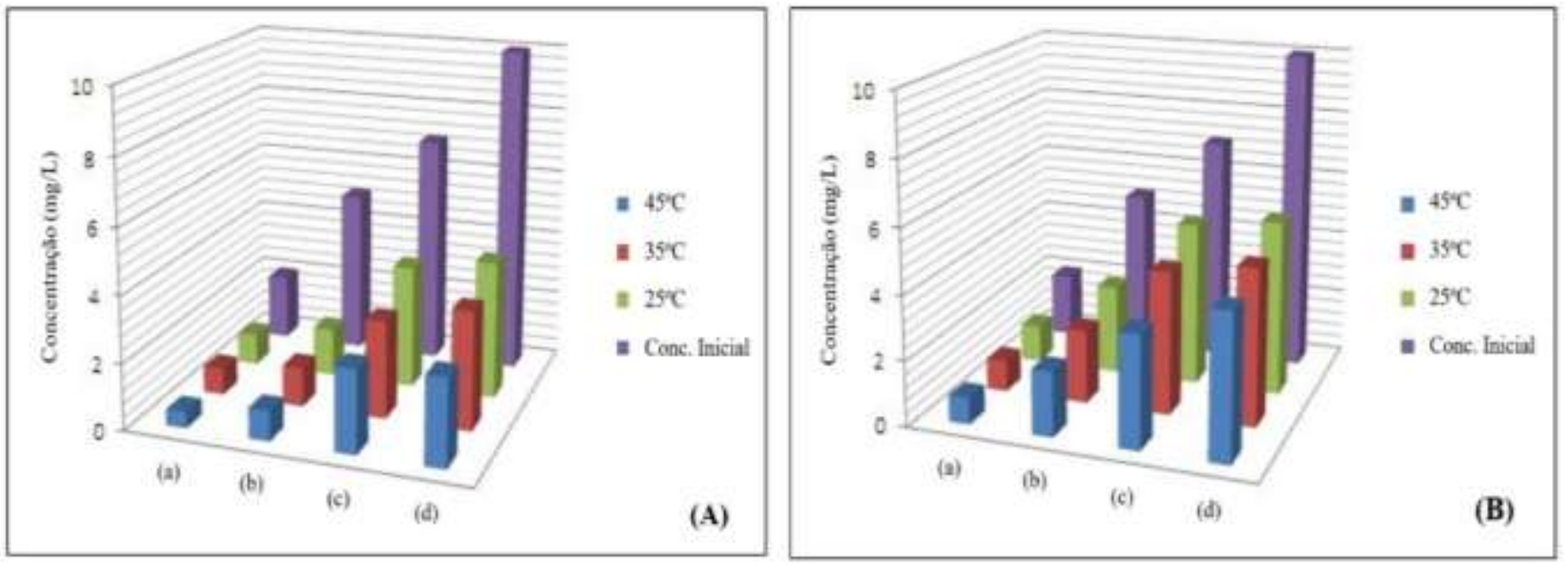

Fonte: Autores.

O aumento da adsorção com a elevação da temperatura indica um processo endotérmico. Essa característica pode estar relacionada à ocorrência da dilatação dos poros dos adsorventes, favorecendo o aumento da capacidade adsortiva dos mesmos. Além disso, o aumento da temperatura eleva o grau de agitação das moléculas de adsorvato, o que pode possibilitar um maior contato com o adsorvente e promover maior adsorção (Müller, Alves, Mondardo, \& Sens, 2019).

\section{Conclusão}

Os resultados mostraram que tanto os ouriços quanto as cascas da amêndoa da Castanha do Brasil podem ser transformados em carvão ativado para serem utilizados com finalidades energéticas. As biomassas apresentaram características favoráveis para este fim, sendo inclusive, semelhantes ou superiores às referenciadas pela literatura.

Conclui-se que o processo de obtenção de carvão ativado a partir das cascas ou dos ouriços da Bertholletia excelsa H.B.K. é bastante satisfatório, pois promove o aproveitamento dos resíduos, e ainda, pode gerar uma forma de renda para os coletores de castanha do Brasil na região Amazônica. Além disso contribuem como uma possível solução na busca por materiais precursores de baixo custo e em quantidades abundantes para a produção de adsorventes.

\section{Agradecimentos}

OS AUTORES AGRADECEM A FUNDAÇÃO DE AMPARO À PESQUISA DO ESTADO DO AMAZONAS (FAPEAM) E A PRÓ-REITORIA DE PESQUISA E PÓS-GRADUAÇÃO (PROPESP/UFAM) PELO APOIO FINANCEIRO PARA A REALIZAÇÃO DESTA PESQUISA.

\section{Referências}

Albadarin, A. B., Collins, M. N., Naushad, M., Shirazian, S., Walker, G., \& Mangwandi, C. (2017). Activated lignin-chitosan extruded blends for efficient adsorption of methylene blue. Chemical Engineering Journal, 307, 264-272.

Associação Brasileira de Normas Técnicas (1986). NBR 8112: Análise imediata de carvão vegetal. ABNT.

Bouvie, L., Borella, D. R., Porto, P. A. O., Silva, A. C., \& Leonel, S. (2016). Caracterização físico-química dos frutos de castanheira do Brasil. Nativa, 4(2), $107-111$.

Brand, M. A. (2010). Energia de Biomassa Florestal: Interciência, 114p. 
Carmona, I. N., Sampaio, J. S., Andrade, F. W. C., Júnior, C. D. C., Junior, S. M. O. M., Moreira, L. S., \& Moutinho, V. H. P. (2017). Potencial Energético da Biomassa e Carvão Vegetal de Resíduos de Castanha-do-Pará (Bertholletia excelsa Bonpl.). In: $3^{\circ}$ Congresso Brasileiro de Ciência e Tecnologia da Madeira: CBCTEM.

Costa, J. R., Castro, A. B. C., Wandelli, E. V., Coral, S. C. T., \& Souza, S. A. G. (2009). Aspectos silviculturais da castanha-do-brasil (Bertholletia excelsa) em sistemas agroflorestais na Amazônia Central. Acta Amazonica, 39(4), 843-850.

Couto, L. C., Couto, L., Watzlawick, L. F., \& Câmara, D. (2004). Vias de valorização energética da biomassa. Biomassa \& Energia, 1(1), 71-92.

Everard, C. D., Mcdonnell, K. P., \& Fagan, C. C. (2012). Prediction of biomass gross calorific values using visible and near infrared spectroscopy. Biomass and Bioenergy, 45, 203-211.

Fernandes, L., \& Souza, C. D. R. (2016). Produção de biodiesel utilizando biomassa regional da Amazônia. In: PIBIC/UFAM - Relatório Final do Programa de Apoio à Iniciação Científica do Amazonas.

Ferreira, E. D. S., Silveira, C. D. S., Lucien, V. G., \& Amaral, A. S. (2009). Caracterização físico-química da amêndoa, torta e composição dos ácidos graxos majoritários do óleo bruto da castanha-do-Brasil (Bertholletia excelsa HBK). Alimentos e Nutrição, 17(2), 203-208.

Ghasemi, M., Mashhadi, S., Asif, M., Tyagi, I., Agarwal, S., \& Gupta, V. K. (2016). Microwave-assisted synthesis of tetraethylenepentamine functionalized activated carbon with high adsorption capacity for Malachite green dye. Journal of Molecular Liquids, 123, 317-325.

Hameed, K. S., Muthirulan, P., \& Meenakshi, S. M. (2017). Adsorption of chromotrope dye onto activated carbons obtained from the seeds of various plants: Equilibrium and kinetics studies. Arabian Journal of Chemistry, 10, S2225-S2233.

Himmelblau, D. M., \& Riggs, J. B. (2014). Engenharia Química: Princípios e Cálculos. (8a ed.,): LTC.

Januszewicz, K., Kazimierski, P., Klein, M., Karda's, D., \& Luczak, J. (2020). Activated Carbon Produced by Pyrolysis of Waste Wood and Straw for Potential Wastewater Adsorption. Materials, 13, 2047.

Klautau, J. V. P. (2008). Análise Experimental de uma Fornalha a lenha de Fluxo Cocorrente Para Secagem de Grãos, Dissertação de Mestrado. PPGERHA/UFPR, Curitiba.

Leandro, R. I. M., Abreu, J. J. C., Martins, C. S., Santos, I. S., Bianchi, M. L., \& Nobre, J. R. C. (2019). Elementary, Chemical and Energy Characteristics of Brazil Nuts Waste (Bertholletia excelsa) in the State of Pará. Floresta e Ambiente, 26 (2), 1-6.

Lillo-Ródenas, M. A., Juan-Juan, J., Cazorla-Amorós, D., \& Linares-Solano, A. (2004). About reactions occurring during chemical activation with hydroxides Carbon, 42(7), 1371-1375.

Marafon, A. C., Santiago, A. D., Amaral, A. F. C., Bierhals, A. N., Paiva, H. L., \& Guimarães, V. S. (2016). Uso da biomassa para a geração de energia. (1ª ed.,): Embrapa Tabuleiros Costeiros, 30p.

Marin, P., Borba, C. E., Módenes, A. N., Oliveira, S. P. D., Passaia, N., \& Figueiredo, L. S. (2015). Avaliação do efeito da temperatura, pH e granulometria do adsorvente na adsorção do corante azul reativo 5G. Engevista, 17(1), 59-68.

Mckendry, P. (2015). Energy production from biomass (part 1): overview of biomass. Bioresource Technology, 83(1), 37-46.

Melo, S. S., Diniz, J. E. M., Guimarães, J. H., Costa, J. S., Brazil, D. S. B., Morais, S. S. S., Brito, D. C., Carvalho, J. T. C., Santos, C. B. R., \& Silva, D. L. (2015). Production and characterization of absorbent heat from the bark of residual Brazil nut bark (Bertholletia excelsa L.). Chemistry Central Journal, 36(9), $1-9$

Müller, L. C., Alves, A. A. A., Mondardo, R. I., \& Sens, M.L. (2019). Adsorção do azul de metileno em serragem de Pinus elliottii (pinus) e Drepanostachyum falcatum (bambu). Engenharia Sanitária e Ambiental, 24(4), 687-695.

Nascimento, V. F. (2012). Caracterização de biomassas amazônicas - ouriço de castanha-do-brasil, ouriço de sapucaia e caroço do fruto do tucumã - visando sua utilização em processos de termoconversão, Dissertação de Mestrado. Faculdade de Engenharia Química/Unicamp, Campinas.

Nobre, J. R. C., Castro, J. P., Motta, J. P., Bianchi, M. L., Trugilho, P. F., Borges, W. M. S., \& Moulin, J. C. (2015). Activated carbon production of the wood residue of the Amazonian area. Scientia Forestalis, 43(108), 895-906.

Nogueira, R. M., Ruffato, S., Carneiro, J. S., Pires, E. M., \& Alvares, V. S. (2014). Avaliação da carbonização do ouriço da castanha-do-brasil em forno tipo tambor. Scientific Electronic Archives, 6, 7-17.

Oliveira, J. M. C., \& Lobo, P. C. (2002). Avaliação do potencial energético de resíduos de biomassa amazônica. In: $4^{\circ}$ Encontro de Energia no Meio Rural, Campinas.

Parikh, J., Channiwala, S. A., \& Ghosal, G. K. (2005). A correlation for calculating HHV from proximate analysis of solid fuels. Fuel, 84(5), 487-494.

Patias S. G. O., Sávio J., Costelli M. C., Silva A. S., Cancelier A., \& Lopes T. J. (2015). Obtenção de carvão adsorvente oriundo da casca de pequi (Caryocar brasiliense) e sua aplicação no tratamento de efluentes da indústria têxtil através do processo de adsorção. Revista Eletrônica em Gestão, Educação e Tecnologia Ambiental, 19(2), 1482-1492.

Pereira, D. T. O., Nobre, J. R. C., \& Bianchi, M. L. (2019). Energy quality of waste from Brazil nut (bertholletia excelsa), in the state of Pará. Brazilian Journal of Development, 5(4), 3258-3265.

Reza, M. S., Yun, C. S., Afroze, S.; Radenahmad, N., Bakar, M. S. A., Saidur, R., Taweekun, J., \& Azad, A. K. (2020). Preparation of activated carbon from biomass and its' applications in water and gas purification, a review. Arab Journal Of Basic And Applied Sciences, 27(1), 208-238. 
Research, Society and Development, v. 10, n. 2, e53310212698, 2021 (CC BY 4.0) | ISSN 2525-3409 | DOI: http://dx.doi.org/10.33448/rsd-v10i2.12698

Saini, J. K., Saini, R., \& Tewari, L. (2015). Lignocellulosic agriculture wastes as biomass feedstocks for second-generation bioethanol production: concepts and recent developments. 3 Biotech, 5(4), 337-353.

Santos, E. C. S., \& Júnior, L. D. (2016). Caracterização química de resíduos agroindustriais e de extrativismo na Amazônia. In: Anais do Congresso Técnico Científico da Engenharia e da Agronomia: CONTECC.

Scatolino, M. V., Cabral, L. F. N., Protásio, T. P., Carneiro, A. C. O., Andrade, C. R., Guimarães Jr, J. B., \& Mendes, L. M. (2018). Options for generation of sustainable energy: production of pellets based on combinations between lignocellulosic biomasses. Waste and Biomass Valorization, 9(3), 479-489.

Shen, J., Zhu, S., Liu, X., Zhang, H., \& Tan, J. (2010). The prediction of elemental composition of biomass based on proximate analysis. Energy Conversion and Management, 51, 983-987.

Souza, R., \& Trindade, A. (2015). A biomassa como solução energética para o estado do Amazonas. In: 10 Congresso sobre Geração Distribuída e Energia no Meio Rural, São Paulo.

Vassilev, S. V., Baxter, D., Vassileva, C. G., \& Andersen, L. K. (2013). An overview of the composition and application of biomass ash. Part 1. Phase-mineral and chemical composition and classification. Fuel, 105, 40-76.

Vieira, A. C. (2013). Caracterização da biomassa proveniente de resíduos agrícolas, Dissertação de Mestrado. Universidade Estadual do Oeste do Paraná, Cascavel.

Yagub, M. T., Sen, T. K., \& Ang, H. M. (2012). Equilibrium, Kinetics, and Thermodynamics of Methylene Blue Adsorption by Pine Tree Leaves. Water Air \& Soil Pollution, 223, 5267-5282.

Sanchez, C. G. (2010). Tecnologia da Gaseificação de Biomassa. Editora: Átomo, 189p. 